\title{
Capture and Visualisation of Radio Signals with an Open Source, Single Chip Spectrum Analyser
}

\author{
David Northcote*, Lewis McLaughlin ${ }^{\dagger}$, Louise H. Crockett and Robert W. Stewart \\ Department of Electronic and Electrical Engineering, University of Strathclyde, Glasgow, Scotland, UK \\ *david.northcote@strath.ac.uk, ${ }^{\dagger}$ lewis.mclaughlin@strath.ac.uk
}

\begin{abstract}
This demonstration will present a single chip spectrum analyser that has been developed using the Xilinx Zynq Radio Frequency System on Chip (RFSoC), and the PYNQ software framework. The design uses the RFSoC's high speed Radio Frequency Analogue to Digital Converters (RF ADCs) to capture $2.048 \mathrm{GHz}$ of instantaneous bandwidth and directly sample signals at frequencies up to $4.096 \mathrm{GHz}$ using higher order Nyquist Zone techniques. All signal processing, software control, and graphical user interface functions are hosted entirely on the same RFSoC chip. As well as presenting the functionality achieved by the spectrum analyser, we will describe its underlying architecture and demonstrate its use in exploring the radio spectrum by showing examples of ambient signals.
\end{abstract}

Index Terms-RFSoC, PYNQ, radio, spectrum analysis

\section{INTRODUCTION}

Spectrum analysis is a commonly required function in applications such as wired and wireless communications, instrumentation, physics and astronomy. However, spectrum analysers can be expensive, particularly for high bandwidth applications sampling at multiple GHz. In this work, we developed a single chip spectrum analyser application that is open source [1], and available for others to investigate and build upon. Using a supported RFSoC development board, the spectrum analyser can be used to analyse ambient radio signals captured using a cheap, off-the-shelf antenna.

The Zynq RFSoC family of devices combine Programmable Logic (PL), a Processing System (PS), and high speed data converters (RF ADCs and RF DACs) [2] which can operate at up to $4.096 \mathrm{GHz}$ (for Gen 1 devices as adopted here). Our spectrum analyser is implemented entirely on the RFSoC chip, with user control and visualisation functionality designed using the PYNQ software framework [3]. In particular, we make use of Python dashboards to present an intuitive and uncluttered graphical interface to the user, which is accessible via a standard web browser.

\section{The Spectrum Analyser Demonstration}

This demonstration will present the RFSoC spectrum analyser tool, which is capable of acquiring $2.048 \mathrm{GHz}$ of instantaneous bandwidth and directly sample signals at frequencies up to $4.096 \mathrm{GHz}$ using the second order Nyquist Zone. Several features will be demonstrated including adaptive bandwidth control, reprogrammable windowing, a flexible Fast Fourier Transform core, and floating-point power spectral density calculations. The design of the spectrum analyser architecture, shown in Figure 1, will also be described.

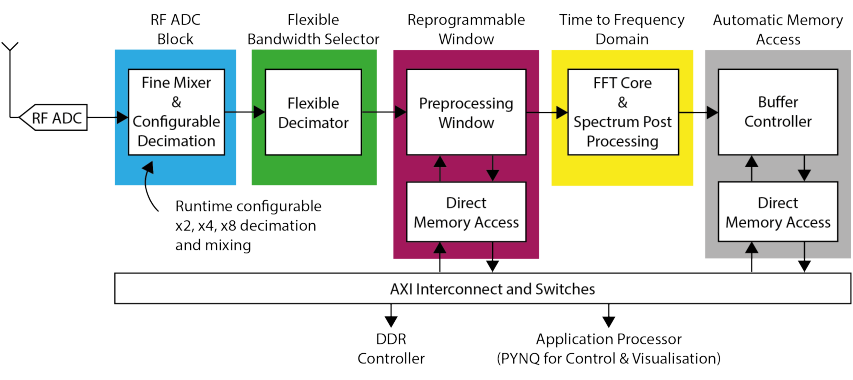

Fig. 1. Architecture overview for one channel of the spectrum analyser

Integrating the spectrum analyser into the PYNQ framework and the design of a graphical interface will be demonstrated using Voila-dashboards [4]. The resulting spectrum analyser tool shown in Figure 2 will be used to explore the radio spectrum, where ambient radio signals including frequency modulated radio waves, digital audio broadcasts, and business radio transmissions can be observed. Furthermore, we will investigate the Wi-Fi spectrum in the second Nyquist Zone, and explore $4 \mathrm{G}$ and $5 \mathrm{G}$ spectrum access bands.

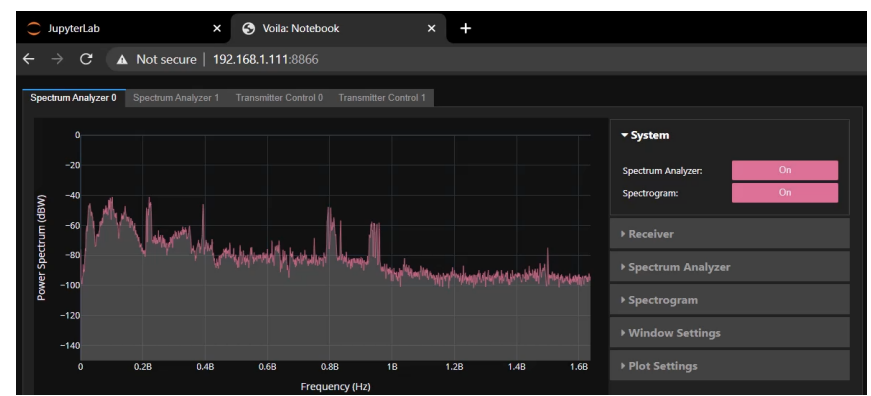

Fig. 2. Dual channel RFSoC spectrum analyser dashboard.

\section{ACKNOWLEDGEMENT}

The authors warmly thank Xilinx Inc. for the support and collaboration that enabled this work.

\section{REFERENCES}

[1] StrathSDR. Spectrum Analyser on PYNQ. Accessed: May. 18, 2021 [Online]. Available: https://github.com/strath-sdr/rfsoc_sam/tree/v0.3.1

[2] Xilinx Inc., Zynq UltraScale+ RFSoC RF Data Converter 2.3, PG269, Jun. 2020.

[3] - Python Productivity on Zynq, PYNQ. Accessed: May. 18, 2021 [Online]. Available: http://www.pynq.io/

[4] Voila-Dashboards. Voila. Accessed: May. 18, 2021. [Online]. Available: https://github.com/voila-dashboards/voila 